\title{
Propriedades mecânicas da madeira resinada de Pinus elliottii
}

\author{
Mechanical properties of Pinus elliottii tapped wood
}

\author{
André Luiz Missio ${ }^{I^{*}}$ Pedro Henrique Gonzalez de Cademartori ${ }^{I I}$ Bruno Dufau Mattos ${ }^{\text {III }}$ \\ Michael Weiler ${ }^{I V}$ Darci Alberto Gatto ${ }^{I}$
}

\section{RESUMO}

\begin{abstract}
As propriedades mecânicas por flexão estática da madeira resinada de Pinus elliottii foram avaliadas por meio de testes destrutivos e não destrutivos. Para tanto, foram confeccionados corpos de prova de $10 \times 10 \times 200 \mathrm{~mm}^{3}$ (radial, tangencial e longitudinal), levando em consideração dois fatores: lenho (juvenil, próximo à medula e adulto, próximo à casca) e resina, isto é, corpos de prova oriundos de toras com e sem estrias de resinagem. Para a avaliação não destrutiva, utilizou-se um aparelho de ultrassom com transdutores do tipo ponto seco, em que se pode calcular a velocidade da onda ultrassônica e consequentemente o módulo de elasticidade dinâmico $\left(E_{D}\right)$. Para obtenção do módulo de elasticidade (MOE) e do módulo de ruptura (MOR), realizou-se o ensaio destrutivo de flexão estática de três pontos. De posse das curvas de força x deformação, calculou-se a fragilidade do material. Os resultados confirmaram que o fator lenho foi significativo para as propriedades analisadas e ofator resina, em especial, para aumento do MOR e da massa especifica. A fragilidade aumentou quando analisada a madeira juvenil e o fator resina quando incluso tendeu a aumentar a fragilidade no lenho adulto. $O$ modelo de regressão múltiplo com variáveis Dummies, proposto para estimar o MOE pelo $E_{D}$, foi significativo em $1 \%$ de probabilidade de erro $\left(r^{2}=0,75\right)$. Os resultados apresentados no presente trabalho mostraram que os fatores lenho e resina não afetaram negativamente as propriedades mecânicas da madeira de Pinus elliottii, em que, para o segundo fator, conclui-se, de maneira geral, que a madeira serrada oriunda de toras resinadas pode não representar danos significativos quando em serviço estrutural.
\end{abstract}

Palavras-chave: Slash Pine, resina de pinus, flexão estática, método não destrutivo, dummies.

\section{ABSTRACT}

The mechanical properties for static bending of Pinus elliottii tapped wood were evaluated by nondestructive

\begin{abstract}
and destructive tests. To achieve this, samples measuring $10 \times 10 \times 200 \mathrm{~mm}^{3}$ (thickness, width and length) were prepared considering two factors: wood (juvenile, near to pith and mature, near to bark) and rosin, i.e., samples from $\operatorname{logs}$ with and without tapping grooves. The nondestructive evaluation were performed using an ultrasound equipment with dry-point transducers in order to measure the ultrasonic velocity and, consequently, the dynamic modulus of elasticity $\left(E_{D}\right)$. The modulus of elasticity (MOE) and the modulus of rupture (MOR) were measured through static bending tests. The wood brittleness were determined through the analysis of force $x$ deformation plots. The results confirmed the significance of wood factor for the properties analyzed and the factor rosin, especially for MOR and density. The brittleness increased for the juvenile wood and, when included the factor rosin, this property tends to increase for the mature wood. The multiple regression model with Dummies variables proposed to estimate MOE as a function of $E_{D}$ was significant at $1 \%$ of probability of error $\left(r^{2}=0.75\right)$. Moreover, the results showed that factor wood and factor rosin did not negatively affect the mechanical properties of Pinus elliotti wood. In general, the timber from tapped logs could not represent significant damages when in structural service.
\end{abstract}

Key words: Slash pine, pine resin, static bending, nondestructive method, dummies.

\section{INTRODUÇÃO}

O gênero Pinus compreende aproximadamente 600 espécies florestais, em que a madeira das espécies Pinus elliottii e Pinus taeda destaca-se por apresentar grande aplicação industrial no Brasil. Essas espécies foram as que melhor se

IPrograma de Pós-graduação em Engenharia Florestal (PPGEF), Centro de Ciências Rurais (CCR), Universidade Federal de Santa Maria (UFSM), 97105-900, Santa Maria, RS, Brasil. E-mail: andreluizmissio@gmail.com. *Autor para correspondência.

IIPrograma de Pós-graduação em Engenharia Florestal (PPGEF), Centro de Ciências Florestais e da Madeira, Universidade Federal do Paraná (UFPR), Curitiba, PR, Brasil.

"IIPrograma de Pós-graduação em Engenharia e Ciência dos Materiais (PIPE), UFPR, Curitiba, PR, Brasil.

${ }^{\mathrm{IV}}$ Divisão de Métodos e Processos, Engenheiro Industrial Madeireiro, S.C.A Mobiliário Contemporâneo, Bento Gonçalves, RS, Brasil. Recebido 08.04.13 Aprovado 10.12.14 Devolvido pelo autor 06.03.15 CR-2013-0475.R1 
adaptaram ao clima na região sul e sudeste do país, tornando-se fontes renováveis de ciclo rápido, com mercado abrangendo os setores de paletes, embalagens, mobiliário, construção civil e indústrias de celulose e papel. Sabe-se também que o ciclo econômico não se restringe apenas à madeira, pois a resinagem de pinus caracteriza-se como uma das mais importantes explorações econômicas desses plantios. De acordo com a Associação dos Resinadores do Brasil (ARESB, 2013), atualmente, a resina é extraída através da abertura de estrias na base da árvore, onde são colocados estimulantes que evitam a cicatrização do local, o que permite a extração por um período mais longo.

Atrás apenas da China no mercado mundial, a produção de resina no Brasil na safra 2012-2013 foi de 96 mil toneladas, em que se estimou o seu preço médio em março de 2013 em R $\$ 1491,82$ por tonelada (ARESB, 2013). A resina é composta pela mistura de terpenoides, o breu (diterpeno, $\mathrm{C}_{20}$ ), a terebintina (monoterpeno, $\mathrm{C}_{10}$ e sesquiterpeno, $\mathrm{C}_{15}$ ) (PHILLIPS \& CROTEAU, 1999; BOHLMANN \& KEELING, 2008) e é utilizada principalmente em produtos químicos intermediários, adesivos, revestimentos (STUBBS et al., 1984), solventes ou agentes de limpeza de tintas, vernizes e na indústria farmacêutica (FAO, 1995).

De acordo com CADEMARTORI et al. (2012), o processo de resinagem é relevante pela sua abrangência em termos de mercado consumidor e escala industrial, já que é considerada como uma forma secundária e rentável de aproveitamento da floresta plantada. Os mesmos autores sugerem que a resinagem pode influenciar diretamente em diversos aspectos qualitativos da madeira, tornando-se assim necessária uma melhor avaliação desse material, para que os seus produtos oriundos possam ganhar mercado e estabelecer um conceito cada vez mais favorável entre os consumidores.

Em geral, a qualidade da madeira serrada de Pinus utilizada em diferentes nichos do mercado nacional e internacional é avaliada visualmente em função da presença de características do material, tais como esmoados, empenamentos, nós e bolsas de resina. O Catálogo de Normas de Madeira Serrada de Pinus (ABPM, 1990) estabelece classes de qualidade para a madeira serrada, as quais indicam que a presença de bolsas de resina afeta negativamente o material. As restrições da norma estabelecem que a madeira de melhor qualidade não deve apresentar bolsas de resina, enquanto as classes inferiores apontam a existência de bolsas, porém com restrições a sua porcentagem em relação à largura ou ao comprimento da peça.
Juntamente com a qualidade da madeira, a avaliação e caracterização das propriedades mecânicas desse material devem ser consideradas como um aditivo de maneira a incrementar ou expandir a utilização da madeira de pinus, dadas as informações concebidas por estas propriedades, essencialmente relacionadas à resistência mecânica a distintos esforços quando em serviço. Nesse contexto, o presente estudou teve por objetivo avaliar as propriedades mecânicas por flexão estática da madeira resinada de Pinus elliottii por meio de técnicas não destrutivas e destrutivas.

\section{MATERIAL E MÉTODOS}

A madeira da espécie Pinus elliottii Engelm. foi proveniente de um plantio homogêneo de 7.200 ha na região litorânea do município de São José do Norte (31 59 '59.40” S, 52 ${ }^{\circ} 1^{\prime} 59.88^{\prime}$ ' O), estado do Rio Grande do Sul - Brasil. O plantio tem espaçamento $2 \times 2 \mathrm{~m}^{2}$, não foi realizado nenhum manejo florestal e as árvores foram resinadas de 10 a 12 vezes antes do abate, sendo um processo por ano, isto é, inicia-se no período de inverno e finaliza-se no período de verão. As árvores possuíam entre 28 e 32 anos.

Foram selecionadas aleatoriamente seis toras, três com estrias de resinagem e três sem estrias de resinagem. As toras foram desdobradas em tábuas tangenciais e radiais de uma polegada. Das tábuas, foram confeccionados corpos de prova de $10 \times 10 \times 200 \mathrm{~mm}^{3}$ (radial, tangencial e longitudinal). Os corpos de prova foram confeccionados considerando dois fatores: lenho juvenil (próximo à medula) e lenho adulto (próximo à casca), e fator resina, isto é, corpos de prova oriundos de toras com e sem estrias de resinagem. Após a etapa de confecção, os corpos de prova foram climatizados em condição de $20^{\circ} \mathrm{C}$ e $65 \%$ de umidade relativa do ar.

Foram realizados dois tipos de avaliação mecânica, a não destrutiva com a utilização de um aparelho de ultrassom e a destrutiva em máquina universal de ensaios. Para avaliação por ultrassom, foram utilizados transdutores ponto seco com $54 \mathrm{kHz}$ de frequência. Foram realizadas três medições no sentido longitudinal dos corpos de prova, pelo método direto, para obtenção da velocidade de propagação da onda ultrassônica (V), em função do comprimento do corpo de prova, sendo utilizada a média aritmética das três medições. Posteriormente, calculou-se o módulo de elasticidade dinâmico $\left(\mathrm{E}_{\mathrm{D}}\right)$, em $\mathrm{MPa}$, pela equação $\mathrm{E}_{\mathrm{D}}=\mathrm{V}^{2} \cdot \rho_{\mathrm{ap}} \cdot 10^{-6}$, em que: $\mathrm{V}=$ velocidade de propagação da onda ultrassônica $\left(\mathrm{m} \mathrm{s}^{-1}\right) ; \rho_{\mathrm{ap}}=$ massa específica aparente na condição climatizada $\left(\mathrm{kg} \mathrm{m}^{-3}\right)$, obtida 
conforme norma da Associação Brasileira de Normas Técnicas (NBR, 1997).

Os ensaios destrutivos de flexão estática foram realizados em uma máquina universal de ensaios (EMIC D30000), com célula de carga de $2 \mathrm{kN}$ e vão de $140 \mathrm{~mm}$, de acordo com os procedimentos normativos da American Society for Testing and Materials D143-94 (ASTM, 2000). Dessa maneira, foi obtido o módulo de elasticidade (MOE) e o módulo de ruptura (MOR).

De posse das curvas de força $\mathrm{x}$ deformação obtidas no ensaio de flexão estática, apropriedade da fragilidade (F) foi calculada através da metodologia proposta por PHUONG et al. (2007), utilizando uma proporção do trabalho absorvido na região elástica $\left(\mathrm{A}_{\mathrm{E}}\right)$ em relação ao trabalho absorvido na região plástica $\left(A_{p}\right)$ do diagrama força $x$ deformação conforme a equação: $F=\left(A_{E} / A_{E}+A_{P}\right)$. De acordo com os mesmos autores, o limite proporcional foi obtido ao desenhar uma curva de correlação linear com coeficiente de determinação de 0,999 , partindo da carga de $0 \%$ até o limite elástico.

A análise estatística procedeu-se com delineamento inteiramente casualizado, com uma espécie (Pinus elliottii), dois tipos de lenho (juvenil e adulto), duas condições (toras com e sem estrias de resinagem) e trinta e sete repetições (corpos de prova). Os dados foram interpretados através de análise de variância (ANOVA) fatorial e criação de modelos matemáticos de regressão. Primeiramente, foram verificadas a normalidade e homogeneidade de variância dos dados, pressupostos antes de qualquer teste paramétrico. $\mathrm{Na}$ ANOVA, foram realizadas comparações de médias, para os fatores lenho e resinagem, pelo teste $\mathrm{F}$ em $5 \%$ de probabilidade de erro. Em caso de rejeição da hipótese nula, foi realizado teste de médias HSD de Tukey, em $5 \%$ de probabilidade de erro.

Os modelos de regressão foram selecionados de acordo com o coeficiente de determinação $\left(\mathrm{r}^{2}\right)$, erro padrão médio $\left(\mathrm{S}_{\mathrm{yx}}\right)$ e estatística F. Para validação do modelo, foi feito teste de heterogeneidade de variância, normalidade e independência dos erros pelos testes White, ShapiroWilk e Durbin-Watson, respectivamente. Foram inseridas no modelo duas variáveis Dummies, D1 e D2, que assumiram valores 0 e 1 para presença ou não dos fatores lenho e resinagem. As variáveis Dummies foram definidas da seguinte forma: $\mathrm{D} 1=0$ se o fator resinagem não estiver presente no $\mathrm{E}_{\mathrm{D}} ; \mathrm{D} 1=1$ se o fator resinagem estiver presente no $E_{D} ; D 2=0$ se o lenho juvenil está presente no $\mathrm{E}_{\mathrm{D}} ; \mathrm{D} 2=1$, se o lenho adulto está presente no $\mathrm{E}_{\mathrm{D}}$. Foi também avaliada a significância no coeficiente angular entre os fatores lenho e resina, em que se criaram as variáveis $\mathrm{X} \cdot \mathrm{D} 1 \mathrm{e}$ $\mathrm{X} \cdot \mathrm{D} 2$. A significância de cada variável independente no modelo foi verificada por meio do teste $\mathrm{T}$ usual, considerando variáveis significativas aquelas que apresentaram p-valor menor que 0,05 $(\mathrm{P}<0,05)$.

\section{RESULTADOS E DISCUSSÃO}

De acordo com a análise de variância fatorial, os fatores lenho e resinagem tiveram um efeito estatisticamente significativo $(\mathrm{P}<0,05)$ para todas as propriedades, com exceção da massa específica $\left(\rho_{\mathrm{ap}}\right)$ para o fator lenho e do módulo de elasticidade (MOE) e da fragilidade $(F)$ para o fator resinagem (Tabela 1). A interação entre os fatores (Lenho $\mathrm{x}$ Resinagem) foi estatisticamente significativa $(\mathrm{P}<0,05)$ para as propriedades da $\rho_{\text {ap }}$, MOE, módulo de elasticidade dinâmico $\left(\mathrm{E}_{\mathrm{D}}\right)$ e fragilidade $(\mathrm{F})$, e não significativa $(\mathrm{P}>0,05)$ para o módulo de ruptura $(\mathrm{MOR})$ e a velocidade de propagação da onda ultrassônica (V).

A $\rho_{\text {ap }}$ se mostrou $14,8 \%$ superior no lenho adulto, quando comparada ao lenho juvenil, para os corpos de prova oriundos de toras sem estrias de resinagem. Quanto maior a proporção de lenho juvenil, menor é a $\rho_{\text {ap }}$ (BALLARIN \& NOGUEIRA, 2005; KRETSCHMANN, 2008; BAL \& BEKTAŞ, 2012). Em toras com a presença de estrias de resinagem, não houve diferenças significativas para a $\rho_{\text {ap }}$ entre os lenhos. Para ambos os tipos de lenho, houve diferença significativa entre a $\rho_{\text {ap }}$ dos corpos de prova oriundos de toras com e sem estrias de resinagem, sendo $61 \%$ superior para o lenho juvenil e 35,7\% para o lenho adulto (Tabela 2). De acordo com o Instituto de Pesquisas e Estudos Florestais (IPEF, 1978), a $\rho$ da resina bruta é de $0,97 \mathrm{~g} \mathrm{~cm}^{-3}$, valor similar ao encontrado para o lenho juvenil oriundo de toras com estrias de resinagem.

Pode-se observar que a resina age como um material de enchimento e se mantém retida nos espaços vazios da madeira. Assim, o incremento da $\rho_{\text {ap }}$ em relação ao fator resinagem é maior no lenho juvenil, devido a esse lenho apresentar maior diâmetro de lúmen e paredes celulares mais finas (BUCUR, 2006) do que o lenho adulto, apresentando assim mais sítios para a resina se manter depositada.

Para o MOE e o MOR, os resultados foram iguais em relação aos fatores lenho e resinagem. No lenho adulto, os valores médios do MOE e do MOR foram 79,8 e 48,9\% superiores ao do lenho juvenil, respectivamente, para corpos de prova provenientes de toras sem estrias de resinagem. Esses resultados se mostraram representativos para a espécie e para a variação que ocorre entre lenhos (ADAMOPOULOS et al., 2007; KRETSCHMANN, 2008). Em toras com 
Tabela 1- Análise de variância fatorial das propriedades da madeira da espécie Pinus elliottii sob influência dos fatores lenho e resinagem.

\begin{tabular}{|c|c|c|c|c|c|}
\hline Propriedade & Fonte & SQ & GL & QM & $\mathrm{F}$ \\
\hline \multirow{4}{*}{$\rho_{\text {ap }}$} & Lenho & 0,0295343 & 1 & 0,0295343 & $3,41^{\mathrm{ns}}$ \\
\hline & Resinagem & 3,25845 & 1 & 3,25845 & $376,67^{* *}$ \\
\hline & Lenho x Resinagem & 0,127247 & 1 & 0,127247 & $14,71^{* *}$ \\
\hline & Resíduos & 1,26299 & 146 & 0,00865062 & \\
\hline \multirow{4}{*}{ MOE } & Lenho & 7,20398E8 & 1 & 7,20398E8 & $112,13^{* *}$ \\
\hline & Resinagem & $1,04553 \mathrm{E} 7$ & 1 & $1,04553 \mathrm{E} 7$ & $1,63^{\mathrm{ns}}$ \\
\hline & Lenho x Resinagem & $3,40136 \mathrm{E} 7$ & 1 & $3,40136 \mathrm{E} 7$ & $5,29^{*}$ \\
\hline & Resíduos & $9,12336 \mathrm{E} 8$ & 142 & $6,4249 \mathrm{E} 6$ & \\
\hline \multirow{4}{*}{ MOR } & Lenho & 36156,1 & 1 & 36156,1 & $117,82^{* *}$ \\
\hline & Resinagem & 1821,84 & 1 & 1821,84 & $5,94^{*}$ \\
\hline & Lenho x Resinagem & 106,642 & 1 & 106,642 & $0,35^{\mathrm{ns}}$ \\
\hline & Resíduos & 44497,8 & 145 & 306,882 & \\
\hline \multirow{4}{*}{$\mathrm{V}$} & Lenho & 2,99887E6 & 1 & 2,99887E6 & $59,52^{* *}$ \\
\hline & Resinagem & $1,74508 \mathrm{E} 6$ & 1 & $1,74508 \mathrm{E} 6$ & $34,64^{* *}$ \\
\hline & Lenho x Resinagem & 328,332 & 1 & 328,332 & $0,01^{\mathrm{ns}}$ \\
\hline & Resíduos & 7,35619E6 & 146 & 50384,8 & \\
\hline \multirow{4}{*}{$\mathrm{E}_{\mathrm{D}}$} & Lenho & $7,27946 \mathrm{E} 7$ & 1 & $7,27946 \mathrm{E} 7$ & $122,40^{* *}$ \\
\hline & Resinagem & $4,54442 \mathrm{E} 7$ & 1 & $4,54442 \mathrm{E} 7$ & $76,41^{* *}$ \\
\hline & Lenho x Resinagem & 2,93391E6 & 1 & 2,93391E6 & $4,93^{*}$ \\
\hline & Resíduos & $8,6831 \mathrm{E} 7$ & 146 & 594733 & \\
\hline \multirow{4}{*}{$\mathrm{F}$} & Lenho & 204,811 & 1 & 204,811 & $16,24^{* *}$ \\
\hline & Resinagem & 3,07788 & 1 & 3,07788 & $0,24^{\mathrm{ns}}$ \\
\hline & Lenho x Resinagem & 129,991 & 1 & 129,991 & $10,31^{* *}$ \\
\hline & Resíduos & & 123 & 12,6138 & \\
\hline
\end{tabular}

Em que: $\rho_{\text {ap }}=$ massa específica $\left(\mathrm{g} \mathrm{cm}^{-3}\right) ; \mathrm{MOE}=$ módulo de elasticidade $(\mathrm{MPa}) ; \mathrm{MOR}=$ módulo de ruptura $(\mathrm{MPa}) ; \mathrm{V}=\mathrm{velocidade}$ de propagação da onda ultrassônica $\left(\mathrm{m} \mathrm{s}^{-1}\right) ; \mathrm{E}_{\mathrm{D}}=$ módulo de elasticidade dinâmico $(\mathrm{MPa}) ; \mathrm{F}=$ fragilidade; $\mathrm{SQ}=\mathrm{soma}$ de quadrados; $\mathrm{GL}=$ graus de liberdade; $\mathrm{QM}=$ quadrado médio. ${ }^{\mathrm{ns}}=$ não significativo; ${ }^{*}=$ significativo em $5 \%$ de probabilidade de erro; ${ }^{* *}=$ significativo em $1 \%$ de probabilidade de erro.

estrias de resinagem, a diferença entre lenhos nos valores médios do MOE e do MOR foram de 42 e $38,8 \%$, respectivamente, inferiores aos observados em toras sem estrias de resinagem.

A V diminuiu nos corpos de prova oriundos de toras com estrias de resinagem, sendo 8,4 e 7,4\% menor no lenho juvenil e adulto, respectivamente, o que evidencia que a velocidade de propagação na resina é menor que na madeira. As ondas de ultrassom são ondas mecânicas que se propagam mais facilmente em meios sólidos, por meio de vibrações moleculares. Dessa forma, a diminuição da V em função do fator resinagem, para ambos os lenhos, pode ser associada à consistência pouco sólida da resina em relação à madeira.

Entre lenhos, os resultados médios apresentados da V foram estatisticamente superiores em aproximadamente $10 \%$ no lenho adulto. Os resultados aqui encontrados foram típicos aos encontrados na literatura para a madeira do gênero Pinus (BALLARIN \& NOGUEIRA, 2005; STANGERLIN et al., 2008). Já $\mathrm{o} \quad \mathrm{E}_{\mathrm{D}}$ apresentou diferenças estatisticamente significativas para ambos os fatores, em especial, o aumento de 35,5 e $14,8 \%$ no $E_{D}$ nos corpos de prova provenientes de toras com estrias de resinagem no lenho juvenil e adulto, respectivamente. Esses resultados são confirmados com o aumento significativo da $\rho_{\text {ap }}$ quando inserido o fator resinagem nos corpos de prova, já que o $\mathrm{E}_{\mathrm{D}}$ é diretamente proporcional a $\rho_{\text {ap }}$ e a V.

Para a F, os corpos de prova oriundos de toras sem estrias de resinagem apresentaram um aumento de $88,5 \%$ no lenho juvenil em comparação com o lenho adulto. Diferentemente do lenho juvenil, os valores médios da F no adulto 
Tabela 2- Valores médios e teste de médias para as propriedades da madeira da espécie Pinus elliottii sob influência dos fatores lenho e resinagem.

\begin{tabular}{|c|c|c|c|}
\hline Propriedade & Lenho & Resinado & Não-resinado \\
\hline \multirow[b]{2}{*}{$\rho_{\text {ap }}$} & Juvenil & $0,936 \mathrm{~b} \mathrm{~A}$ & 0,581 a $A$ \\
\hline & Adulto & $0,905 \mathrm{~b} \mathrm{~A}$ & 0,667 a B \\
\hline \multirow{2}{*}{ MOE } & Juvenil & 8333,31 b A & 6822,32 a A \\
\hline & Adulto & 11834,80 a B & 12267,90 a B \\
\hline \multirow{2}{*}{ MOR } & Juvenil & $76,3285 \mathrm{~b} \mathrm{~A}$ & 67,5928 a A \\
\hline & Adulto & 105,962 a B & 100,63 a B \\
\hline \multirow{2}{*}{$\mathrm{V}$} & Juvenil & 2384,99 a A & 2604,87 b A \\
\hline & Adulto & 2672,30 a B & 2886,23 b B \\
\hline \multirow{2}{*}{$\mathrm{E}_{\mathrm{D}}$} & Juvenil & $5302,17 \mathrm{~b} \mathrm{~A}$ & 3914,08 a A \\
\hline & Adulto & 6421,81 b B & 5596,20 a B \\
\hline \multirow{2}{*}{ Fragilidade } & Juvenil & 8,062 a A & 9,785 a B \\
\hline & Adulto & $7,542 \mathrm{~b} \mathrm{~A}$ & 5,191 a A \\
\hline
\end{tabular}

Em que: $\rho_{\text {ap }}=$ massa específica $\left(\mathrm{g} \mathrm{cm}^{-3}\right) ; \mathrm{MOE}=$ módulo de elasticidade $(\mathrm{MPa}) ; \mathrm{MOR}=$ módulo de $\operatorname{ruptura}(\mathrm{MPa}) ; \mathrm{V}=$ velocidade de propagação da onda ultrassônica $\left(\mathrm{m} \mathrm{s}^{-1}\right) ; \mathrm{E}_{\mathrm{D}}=$ módulo de elasticidade dinâmico (MPa); $\mathrm{F}=$ fragilidade. Médias seguidas pelas mesmas letras, minúsculas na linha e letras maiúsculas na coluna, não apresentam significância a $5 \%$ de probabilidade de erro (Tukey Test, $\mathrm{P}>0,05$ ).

apresentaram um aumento significativo de 45,3\%, quando incluído o fator resinagem nos corpos de prova. A F apresentou uma correlação linear negativa de $-0,52$ com o MOR e -0,50 com o MOE, em que, quanto maior a $\mathrm{F}$, menores os valores do MOR e do MOE, resultado já esperado.

Observou-se que o percentual de fragilidade na fratura do corpo de prova $(\mathrm{F})$ foi uma variável pouco sensível em relação ao tipo de lenho e também em função de corpos de prova oriundos de toras com ou sem estrias de resinagem na madeira de Pinus elliottii. Todavia, para todas as situações (lenhos x resinagem), os valores de $\mathrm{F}$ podem ser considerados baixos (menor que $10 \%$ ), evidenciando que a madeira dessa espécie tem a característica de não romper de maneira frágil, mesmo com a presença de resina.

Para a estimativa do MOE em função do $E_{D}$, do fator resina (D1), do fator lenho (D2), do $E_{D} \cdot D 1$ e do $E_{D} \cdot D 2$, verificou-se que todas as variáveis independentes foram significativas em $1 \%$ de probabilidade de erro quando inseridas no modelo, exceto a variável D1 (dummy 1) que não foi significativa, sendo retirada do modelo máximo final (Tabela 3). A variável do $E_{D}$ foi transformada na variável $\mathrm{X}\left(1 / \mathrm{E}_{\mathrm{D}}\right)$, evitando assim a autocorrelação dos resíduos, detectada através do teste Durbin-Watson.

O modelo final obtido foi: $\mathrm{MOE}=18129,4$ $+5805,24 \cdot \mathrm{D} 2-4,23462 \mathrm{E} 7 \cdot \mathrm{X}-1,02755 \mathrm{E} 7 \cdot \mathrm{X} \cdot \mathrm{D} 1$ $2,33595 \mathrm{E} 7 \cdot \mathrm{X} \cdot \mathrm{D} 2 ; \operatorname{com~R}_{\mathrm{aj}}{ }^{2}=0,747 \mathrm{e} \mathrm{S}_{\mathrm{yx}}=1731,66 \mathrm{MPa}$, isto é, o modelo linear múltiplo proposto explica $74,70 \%$ da variabilidade do MOE. Note que, para um mesmo valor das outras covariáveis, o sinal positivo apresentado na variável D2 (dummy2) indicou que a presença de lenho adulto afetou positivamente o MOE, fato já esperado e reportado por outros autores (BALLARIN \& NOGUEIRA, 2005; GULER et al., 2007; KRETSCHMANN, 2008; STANGERLIN et al., 2008).

Os valores calculados a partir do modelo proposto para cada situação, isto é, juvenil (D1 $=0$; $\mathrm{D} 2=0)$, juvenil + resinagem $(\mathrm{D} 1=1 ; \mathrm{D} 2=0)$, adulto $(\mathrm{D} 1=0 ; \mathrm{D} 2=1)$ e adulto + resinagem $(\mathrm{D} 1=1 ; \mathrm{D} 2=1)$ pode ser visualizado na figura 1 . Observa-se que a

Tabela 3-Regressão linear múltipla e análise de variância para o modelo proposto com variáveis dummies, para o lenho juvenil e adulto com e sem estrias de resinagem de Pinus elliottii.

\begin{tabular}{|c|c|c|c|c|c|}
\hline Parâmetro & Estimação & Erro médio & Estatística T & \multicolumn{2}{|c|}{$\mathrm{P}$} \\
\hline Intercepto & 18129,4 & 1028,53 & 17,6265 & \multicolumn{2}{|c|}{0,00001} \\
\hline D2 & 5805,24 & 1456,82 & 3,98487 & \multicolumn{2}{|c|}{0,00010} \\
\hline $\mathrm{X}$ & $-4,23462 \mathrm{E} 7$ & 3,99212E6 & $-10,6075$ & \multicolumn{2}{|c|}{0,00001} \\
\hline$X \cdot D 1$ & $-1,02755 \mathrm{E} 7$ & $1,85033 \mathrm{E} 6$ & $-5,55332$ & \multicolumn{2}{|c|}{0,00001} \\
\hline $\mathrm{X} \cdot \mathrm{D} 2$ & $-2,33595 \mathrm{E} 7$ & 7,6019E6 & $-3,07284$ & \multicolumn{2}{|c|}{0,0025} \\
\hline Fonte & SQ & GL & QM & $\mathrm{F}$ & $\mathrm{P}$ \\
\hline Modelo & 1,29017E9 & 4 & $3,22542 \mathrm{E} 8$ & 107,56 & 0,00001 \\
\hline Resíduo & $4,19813 \mathrm{E} 8$ & 140 & 2,99866E6 & -- & -- \\
\hline Total & 1,70998E9 & 144 & -- & -- & -- \\
\hline
\end{tabular}

Em que: $\mathrm{SQ}=$ soma de quadrados; $\mathrm{GL}=$ graus de liberdade; $\mathrm{QM}=$ quadrado médio. 


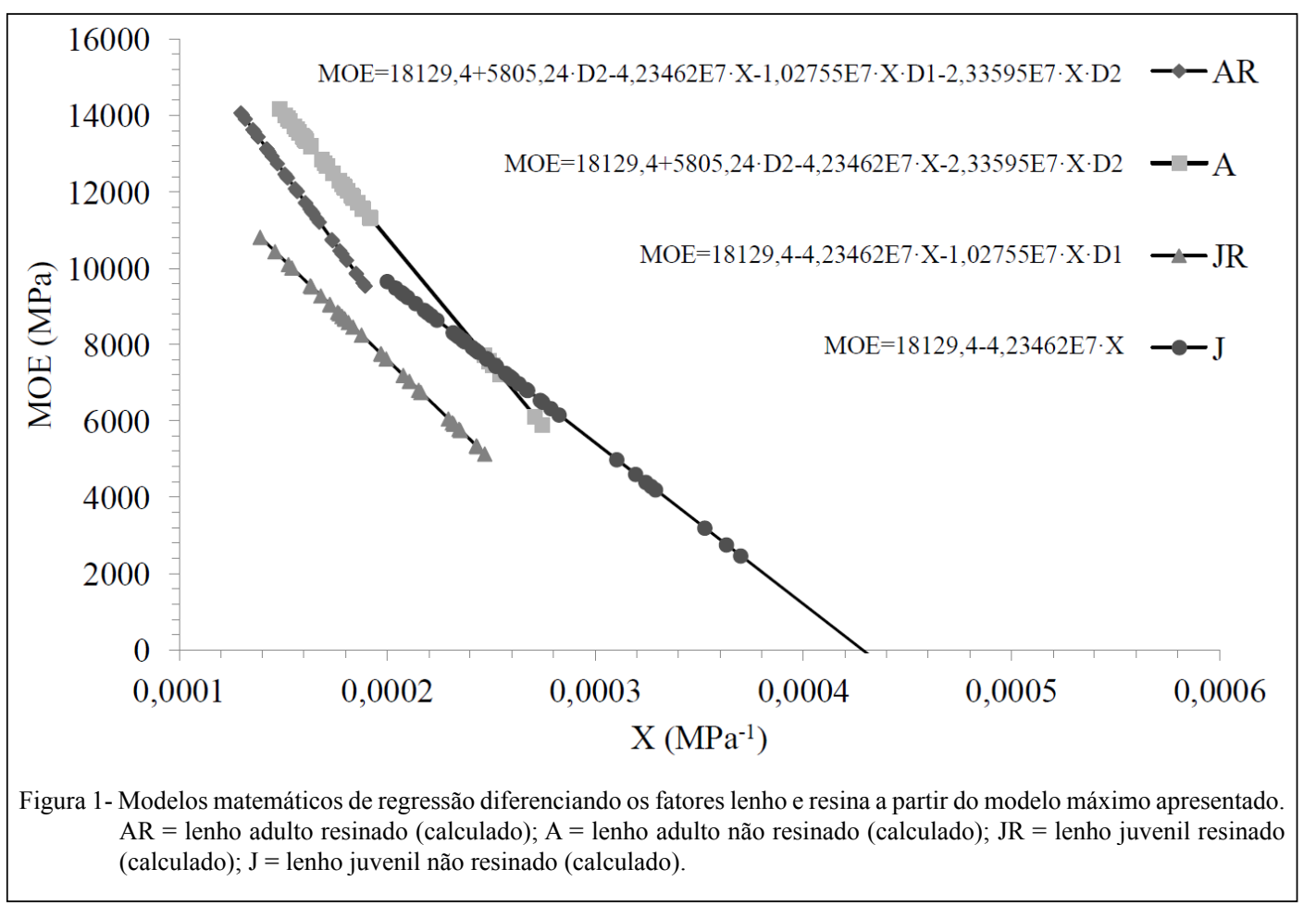

inclusão ou não das variáveis Dummies no modelo alteraram o nível, isto é, o valor do intercepto, e a angulação da reta do modelo de regressão, quando associadas com a variável $X$. Já que as variáveis $\mathrm{X} \cdot \mathrm{D} 1$ e $\mathrm{X} \cdot \mathrm{D} 2$ foram significativas, todas as situações apresentaram um coeficiente angular diferente, mostrando assim que a estimativa do MOE não foi igualmente proporcional, quando os fatores lenho e resina estavam presente no $\mathrm{E}_{\mathrm{D}}$.

\section{CONCLUSÃO}

Os fatores lenho e resinagem influenciaram na massa específica, no módulo de elasticidade, no módulo de ruptura e no módulo de elasticidade dinâmico, em que, no geral, houve acréscimos nos valores médios dessas propriedades. A velocidade de propagação da onda ultrassônica apresentou comportamento distinto entre os fatores, diminuindo com a presença do fator resina.

Para todo fatorial, a fragilidade apresentou valores baixos e não mostrou um comportamento específico em relação aos fatores lenho e resinagem.

O modelo de regressão múltiplo com variáveis Dummies mostrou que os fatores lenho e resinagem afetaram o coeficiente angular da reta de regressão. $\mathrm{O}$ modelo foi significativo em $1 \%$ de probabilidade de erro, sendo assim bom estimador do módulo de elasticidade para cada uma das situações propostas no artigo.

\section{REFERÊNCIAS}

ADAMOPOULOS, S. et al. Strength properties of juvenile and mature wood in black locust (Robinia pseudoacacia L.).Wood and Fiber Science, v.39, n.2, p.241-249, 2007. Disponível em: $<$ http://www.teilar.gr/dbData/Dimosieyseis/Wood\%20and\%20 Fiber\%20Science_2007.pdf $>$. Acesso em 15 mar. 2013.

ASSOCIAÇÃO BRASILEIRA DE PRODUTORES DE MADEIRAS. Catálogo de normas de madeira serrada de pinus. Caxias do Sul: Spectrum Comunicação. 1990. 34p.

ASSOCIAÇÃO DOS RESINADORES DO BRASIL (ARESB). Estatísticas gerais. 2013. Disponível em: $<\mathrm{http}: / / \mathrm{www}$.aresb.com. br/>. Acesso em: mar. 2013

AMERICAN SOCIETY FOR TESTING AND MATERIALS (ASTM). Standards methods of testing small clear specimens of timber: D143-94. Annual Book of ASTM Standard. West Conshohocken, 2000. 31p.

ASSOCIAÇÃO BRASILEIRA DE NORMAS TÉCNICAS (ABNT). Projeto de estruturas de madeira: NBR 7190. Rio de Janeiro, 1997.107p.

BAL, B.C.; BEKTAŞ, İ. The effects of heat treatment on the physical properties of juvenile wood and mature wood of Eucalyptus grandis. Bio Resources, v.7, n.4, p.5117-5127, 2012. Disponível em: <http://ojs.cnr.ncsu.edu/index.php/BioRes/ article/view/BioRes_07_4_5117_Bal_Bektas_Heat_Treatment Eucalyptus_Grandis/ $/ 752>$.Acesso em: fev. 2013. 
BALLARIN, A.W.; NOGUEIRA, M. Determinação do módulo de elasticidade da madeira juvenil e adulta de Pinus taeda por ultrasom. Engenharia Agrícola, v.25, n.1, p.19-28, 2005. Disponível em: <http://www.scielo.br/pdf/eagri/v25n1/24867.pdf $>$. Acesso em: mar. 2013

BOHLMANN, J.; KEELING, C. Terpenoid biomaterials. Plant Journal, v.54, n.4, p.656-669, 2008. Disponível em: <http:// onlinelibrary.wiley.com/doi/10.1111/j.1365-313X.2008.03449.x/ pdf>. Acesso em: mar de 2013. doi: 10.1111/j.1365313X.2008.03449.x

BUCUR, V. Acoustics of wood. Berlin: Springer-Verlag, 2006. 412p. Disponível em: <http://link.springer.com/ book/10.1007\%2F3-540-30594-7>. Acesso em: fev de 2013.

CADEMARTORI, P.H.G.D. et al. Qualidade da madeira serrada de Pinus elliottii Engelm. procedentes de florestas resinadas. Cerne, v.18, n.4, p.577-583, 2012. Disponível em: <http://www.scielo.br/ pdf/cerne/v18n4/v18n4a07.pdf>. Acesso em: mar de 2013. doi: 10.1590/S0104-77602012000400007.

FOOD AND AGRICULTURE ORGANIZATION OF THE UNITED NATIONS (FAO). Flavor and fragrances of plant origin (Non-Wood Forest Products 1). 1995. Disponível em: $<$ http://www.fao.org/docrep/V5350e/V5350e00.htm>. Acesso em: mar de 2013

GULER, C. et al. Some chemical, physical and mechanical properties of juvenile wood from Black Pine (Pinus nigra Arnold) plantations.Journal of Applied Science, v.7, n.5, p.755758, 2007. Disponível em: <http://www.scialert.net/abstract/?d oi=jas.2007.755.758>. Acesso em: mar de 2013. doi: 10.3923/ jas.2007.755.758.

INSTITUTO DE PESQUISAS E ESTUDOS FLORESTAIS (IPEF). O Pinus elliottii, a goma resina e seus derivados. Resina de pinus implantados no Brasil. Piracicaba: ESALQ-USP, Seção de Química, Celulose e Papel, 1978.22p.

KRETSCHMANN, D.E. The influence of juvenile wood content on shear parallel, compression, and tension perpendicular to grain strength and mode I fracture toughness of loblolly pine at various ring orientation. Forest Product Journal, v.58, n.7/8, p.89-96, 2008. Disponível em: <http://www.fpl.fs.fed.us/documnts/fplrp/ fpl_rp647.pdf $>$. Acesso em: fev de 2013.

PHILliPS, M.A.; CROTEAU, R.B. Resin-based defenses in conifers. Trends in Plant Science, v.4, n.5, p.184-190, 1999. Disponível em: <http://www.sciencedirect.com/science/article/ pii/S1360138599014016>. Acesso em: fev de 2013. doi: 10.1016/ S1360-1385(99)01401-6.

PHUONG, L. et al. Effects of heat treatment on brittleness of Styrax tonkinensis wood. Journal of Wood Science, v.53, n.3, p.181-186, 2007. Disponível em: <http://link.springer.com/article /10.1007\%2Fs10086-006-0841-0>. Acesso em: fev de 2013. doi: 10.1007/s10086-006-0841-0.

STANGERLIN, D.M. et al. Determinação do módulo de elasticidade em madeiras por meio de métodos destrutivo e nãodestrutivo. Revista Brasileira de Ciências Agrárias, v.3, n.2, p.145-150, 2008. Disponível em: <http://www.agraria.pro.br/ sistema/index.php?journal $=$ agraria\&page $=$ article $\& o p=$ view $\&$ pat h\%5B $\% 5 \mathrm{D}=284 \&$ path $\% 5 \mathrm{~B} \% 5 \mathrm{D}=164>$. Acesso em: fev de 2013. doi: 10.5039/agraria.v3i2a284.

STUBBS, J. et al. Chemical Stimulation of lightwood in southern pines. Asheville: United States Department of Agriculture, Forest Service, SoutheasternForest Experiment Station, 1984. Disponível em: <http://www.srs.fs.usda.gov/pubs/gtr/gtr_se025.pdf>. Acesso em: mar. 2013. 